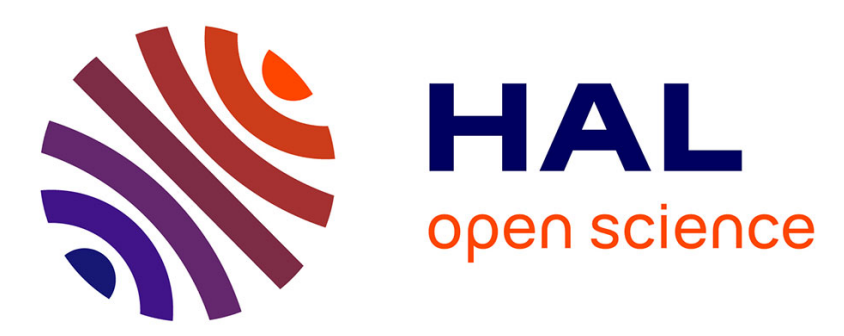

\title{
Variation with Longitude of Aerosol Radioactivity over the Ariantic Ocean
}

Ba Cuong A Nguyen, Gérard Lambert, Bénédicte Ardouin, G Cuong, R D •p•

\section{To cite this version:}

Ba Cuong A Nguyen, Gérard Lambert, Bénédicte Ardouin, G Cuong, R D •p•. Variation with Longitude of Aerosol Radioactivity over the Ariantic Ocean. Journal of Geophysical Research, 1973, 78 (27), pp.6377-6380. 10.1029/JC078i027p06377 . hal-03406756

\section{HAL Id: hal-03406756 https://hal.science/hal-03406756}

Submitted on 28 Oct 2021

HAL is a multi-disciplinary open access archive for the deposit and dissemination of scientific research documents, whether they are published or not. The documents may come from teaching and research institutions in France or abroad, or from public or private research centers.
L'archive ouverte pluridisciplinaire HAL, est destinée au dépôt et à la diffusion de documents scientifiques de niveau recherche, publiés ou non, émanant des établissements d'enseignement et de recherche français ou étrangers, des laboratoires publics ou privés. 


\title{
Variation with Longitude of Aerosol Radioactivity over the Atlantic Ocean
}

\author{
Nguyen Ba Cuong, Gérard Lambert, ${ }^{1}$ and Bénédicte Ardouin \\ Centre des Faibles Radioactivités, Laboratoire Mixte CNRS-CEA \\ 91190-Gif-sur-Y vette, France
}

\begin{abstract}
Aerosols in surface air over the North Atlantic Ocean were collected on air filters on board J. Charcot in September and October 1969 and France during about five months in 1970. The longitudinal variations of fission products and ${ }^{\top} \mathrm{Be}$ concentrations confirm the existence on the earth's surface of 'hot spots' where fallout is very important.
\end{abstract}

In an earlier paper [Nguyen Ba Cuong and Lambert, 1970] it was shown that the radioactive fallout rate varies as a function of not only the latitude but also the longitude of the sampling place. These geographical inhomogeneities could explain the discrepancies sometimes observed when the ${ }^{80} \mathrm{Sr}$ accumulation over continental areas is compared with data obtained over the oceans even at the same latitude. In the paper mentioned above, the study of data published by HASL (Health and Safety Laboratory) enabled us to emphasize the existence of five peaks of ${ }^{\circ r}$ deposition in the vicinity of $0^{\circ}, 80^{\circ}$, and $150^{\circ}$ east and west between $30^{\circ}$ and $60^{\circ} \mathrm{N}$. The phenomenon was attributed to the effect of the jet stream standing waves described by Rossby [1947].

However, it was recognized in our study that a great difficulty was caused by the very poor number of sampling oceanic stations. On the other hand, the radioactive fallout depends highly on the cloud systems.

We have therefore undertaken the measurement of artificial radioactive aerosol concentrations in the lower atmosphere on board oceanographic ship Jean Charcot during a crossing of the North Atlantic Ocean (September-October 1969), then more systematically aboard France during the trips between Le Havre and New York from May to October 1970, i.e., just before the Chinese thermonuclear test of October 14,1970 . The preceding nuclear test was

\footnotetext{
${ }^{1}$ Also with the Université de Picardie.

Copyright (C) 1973 by the American Geophysical Union.
}

made on September 29, 1969: therefore it can be ascertained that nuclear debris collected at sea level 8 months later had a stratospheric origin.

\section{Experimental Procedure}

Aerosols were collected every 24 hours on a 50- by $50-\mathrm{cm}$ Poelmann-Schneider blue filter. The flow of the turbine was $250 \mathrm{~m}^{\mathrm{a}} \mathrm{h}^{-1}$. After about 2 weeks these filters were burned and measured by $\gamma$ spectrometry by means of a $\mathrm{Ge}-\mathrm{Li}$ junction detector (resolution of $3 \mathrm{kev}$ ) and a 4000-channel analyzer. It was therefore possible to distinguish the emitters of very close energy such as ${ }^{7} \mathrm{Be}(477 \mathrm{kev})$ and ${ }^{108} \mathrm{Ru}$ (498 kev) (see Figure 1).

\section{Results}

Figure 2 shows the trips of Jean Charcot from Saint John, Canada, to Brest, France, during the whole period from September 21 to October 28, 1969, and of France between Le Havre, France, and New York from April to October 1970. Data were obtained on board France during trips in both directions. Details of the results are shown in Table 1. The errors indicated in this table correspond to $2 \sigma$ (i.e., associated risk of 5\% for Poisson's law) and are between $\pm 3 \%$ for ${ }^{\mathrm{I}} \mathrm{Ce}$ and $\pm 10 \%$ for ${ }^{\mathrm{T}} \mathrm{Be}$, ${ }^{187} \mathrm{Cs}$, and ${ }^{86} \mathrm{Zr}$.

Figure 3 shows the mean values of the atmospheric concentrations of fission products and ${ }^{7} \mathrm{Be}$ for two different seasons (May to July and August to October) in the five following longitude bands: $1^{\circ}-15^{\circ} \mathrm{W}, 15^{\circ}-30^{\circ} \mathrm{W}, 30^{\circ}-45^{\circ} \mathrm{W}$, $45^{\circ}-60^{\circ} \mathrm{W}$, and $60^{\circ}-74^{\circ} \mathrm{W}$. In both periods, there is a minimum of fission products and ${ }^{~} \mathrm{Be}$ 


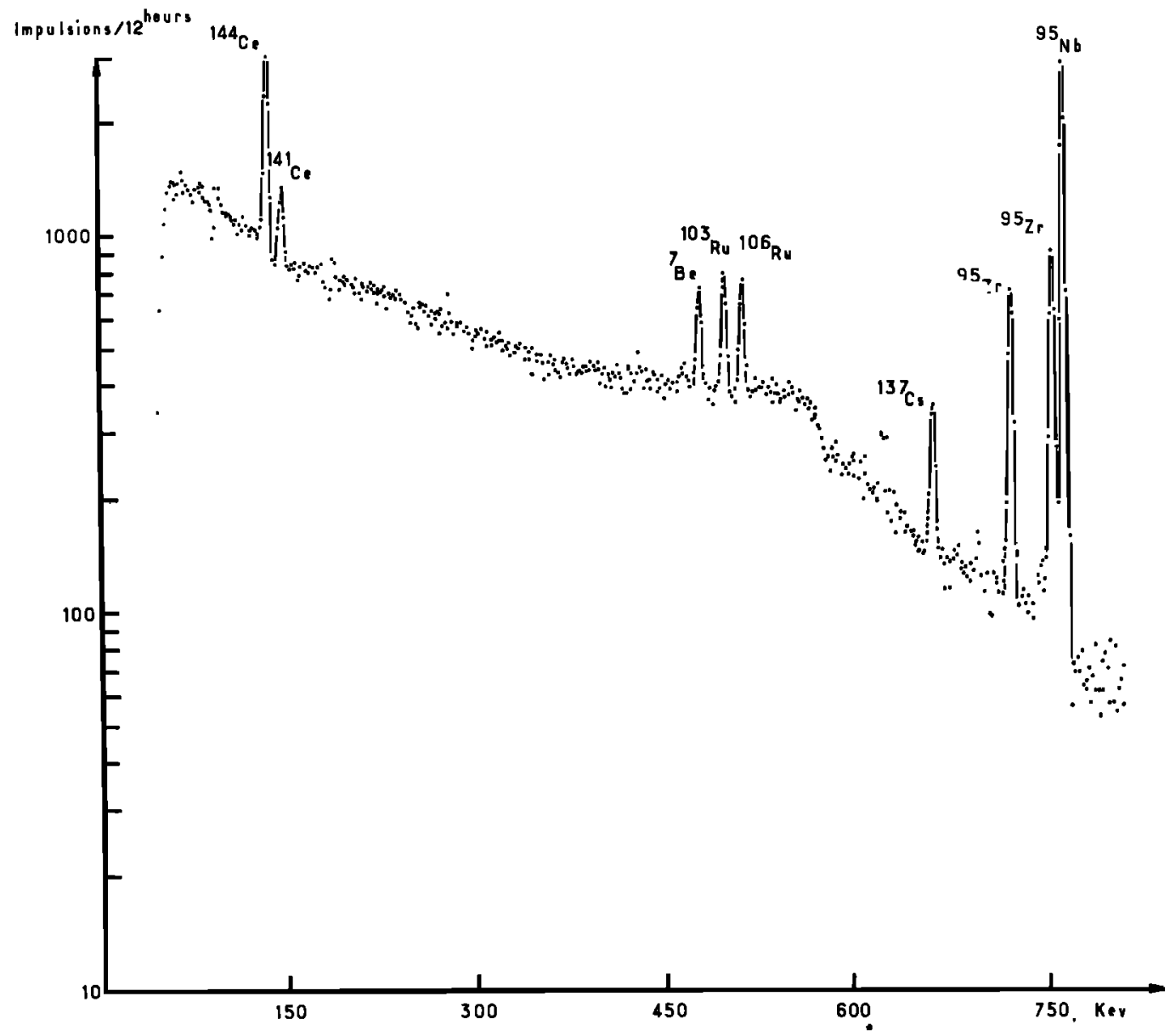

Fig. 1. Gamma spectrum. Collection date: from June 2, 1970 (1200) to June 3, 1970 (1200). Ship's position : $41^{\circ} 51^{\prime} \mathrm{N}, 62^{\circ} 31^{\prime} \mathrm{W}$ at the start; $40^{\circ} 27, \mathrm{~N}, 73^{\circ} 50^{\circ} \mathrm{W}$ at the finish.

near the French coast and two peaks, one on the eastern coast of the United States (from 1.8 to 3 times higher) and the other at about $20^{\circ} \mathrm{W}$ (from 1 to 1.8 times higher).

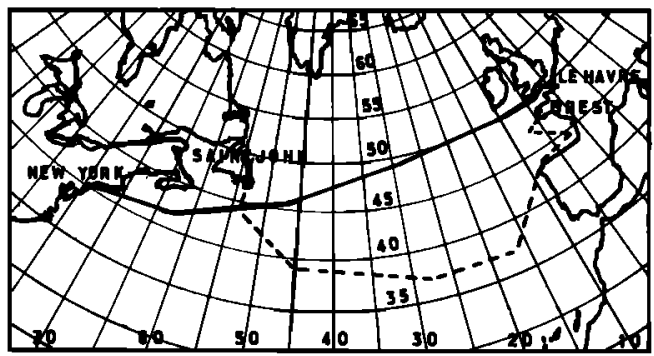

Fig. 2. Trips of oceanographic ships Jean Charcot (dashed line) and France (solid line).
Data from HASL [Volchok and Krajewski, 1972] on fallout at the stations near $80^{\circ} \mathrm{W}$ confirm that the well-known latitudinal effect (about 30\% between New York and Moosonee in 1964 and 1965) cannot entirely explain such a maximum.

The fact that the ratios between ${ }^{\mathrm{B}} \mathrm{Be}$ and fission products are higher at both minimums of radioactivity (Figure 3 ) shows a not negligible contribution of tropospheric ${ }^{\mathrm{B}} \mathrm{Be}$.

\section{Conclusion}

First, it is quite remarkable to find a systematic clear variation in the concentration of artificial radioactive aerosols in places less than $1000 \mathrm{~km}$ apart. Such a trip is made by an air mass in about 2 days. This confirms the very 
short lifetime of 1 day for atmospheric aerosols already measured in the southern hemisphere [Lambert et al., 1972] at low altitude over oceanic areas.

Hence the observed variations of those con- centrations clearly confirm the existence of hot spots at the earth's surface. The maximum concentrations observed at $70^{\circ}$ and $20^{\circ} \mathrm{W}$ coincide rather well with the areas where we had previously found a higher radioactive fallout.

TABLE 1. Concentrations of Radioactive Aerosols over the Atlantic Ocean*

\begin{tabular}{|c|c|c|c|c|c|c|}
\hline \multirow[b]{2}{*}{ Collection Date } & \multicolumn{2}{|c|}{ Position } & \multicolumn{4}{|c|}{ Concentration, $\mathrm{pC} / 10^{3} \mathrm{~m}^{3}$} \\
\hline & Start & Finish & ${ }^{137} \mathrm{Cs}$ & ${ }^{144} \mathrm{Ce}$ & $95_{Z \mathbf{r}}$ & ${ }^{7} \mathrm{Be}$ \\
\hline $\begin{array}{l}\text { Sept. } 21 \text { to } 22,1969 \\
\text { Sept. } 25 \text { to } 26 \\
\text { Sept. } 30 \text { to Oct. } 1 \\
\text { Oct. } 2 \text { to } 3 \\
\text { Oct. } 6 \text { to } 7 \\
\text { Oct. } 8 \text { to } 9 \\
\text { Oct. } 20 \text { to } 21 \\
\text { Oct. } 27 \text { to } 28 \\
\text { Oct. } 31 \text { to Nov. } 1\end{array}$ & $\begin{array}{l}45^{\circ} 37^{\prime} \mathrm{N}, 55^{\circ} 29^{\prime} \mathrm{W} \\
39^{\circ} 35^{\prime} \mathrm{N}, 46^{\circ} 44^{\prime} \mathrm{W} \\
38^{\circ} 15^{\prime} \mathrm{N}, 36^{\circ} 05^{\prime} \mathrm{W} \\
37^{\circ} 21^{\prime} \mathrm{N}, 29^{\circ} 55^{\prime} \mathrm{W} \\
37^{\circ} 56^{\prime} \mathrm{N}, 26^{\circ} 11^{\prime} \mathrm{W} \\
37^{\circ} 33^{\prime} \mathrm{N}, 17^{\circ} 02^{\prime} \mathrm{W} \\
41^{\circ} 51^{\prime} \mathrm{N}, 10^{\circ} 27^{\prime} \mathrm{W} \\
43^{\circ} 46^{\prime} \mathrm{N}, 03^{\circ} 52^{\prime} \mathrm{W} \\
46^{\circ} 54^{\prime} \mathrm{N}, 09^{\circ} 21^{\prime} \mathrm{W}\end{array}$ & $\begin{array}{l}43^{\circ} 37^{\prime} \mathrm{N}, 53^{\circ} 01^{\prime} \mathrm{W} \\
38^{\circ} 34^{\prime} \mathrm{N}, 45^{\circ} 20^{\prime} \mathrm{W} \\
38^{\circ} 02^{\prime} \mathrm{N}, 33^{\circ} 37^{\prime} \mathrm{W} \\
36^{\circ} 47^{\prime} \mathrm{N}, 27^{\circ} 10^{\prime} \mathrm{W} \\
37^{\circ} 53^{\prime} \mathrm{N}, 21^{\circ} 02^{\prime} \mathrm{W} \\
36^{\circ} 40^{\prime} \mathrm{N}, 14^{\circ} 14^{\prime} \mathrm{W} \\
43^{\circ} 46^{\prime} \mathrm{N}, 08^{\circ} 54^{\prime} \mathrm{W} \\
44^{\circ} 35^{\prime} \mathrm{N}, 03^{\circ} 54^{\prime} \mathrm{W} \\
47^{\circ} 41^{\prime} \mathrm{N}, 08^{\circ} 09^{\prime} \mathrm{W}\end{array}$ & $\begin{array}{l}2.6 \pm 0.3 \\
2.5 \pm 0.3 \\
2.6 \pm 0.3 \\
1.3 \pm 0.1 \\
3.2 \pm 0.3 \\
2.1 \pm 0.2\end{array}$ & $\begin{array}{r}29.5 \pm 2.1 \\
17.1 \pm 1.2 \\
6.2 \pm 0.4 \\
23.9 \pm 1.7 \\
10.9 \pm 0.8 \\
10.7 \pm 0.7 \\
20.1 \pm 1.4 \\
18.4 \pm 1.3\end{array}$ & $\begin{aligned} 16.7 & \pm 1.7 \\
7.9 & \pm 0.8 \\
1.5 & \pm 0.2 \\
13.8 & \pm 1.4 \\
6.7 & \pm 0.7 \\
4.8 & \pm 0.5 \\
14.2 & \pm 1.4 \\
7.2 & \pm 0.7 \\
6.9 & \pm 0.7\end{aligned}$ & $\begin{array}{l}50.5 \pm 5.1 \\
22.4 \pm 2.2 \\
17.9 \pm 1.8 \\
58.8 \pm 5.9 \\
34.4 \pm 3.4 \\
67.6 \pm 6.8 \\
47.9 \pm 4.8 \\
47.9 \pm 4.8\end{array}$ \\
\hline $\begin{array}{l}\text { May } 25 \text { to } 26,1970 \\
\text { May } 30 \text { to } 31 \\
\text { May } 31 \text { to June } 1 \\
\text { June } 1 \text { to } 2 \\
\text { June } 2 \text { to } 3 \\
\text { June } 4 \text { to } 5 \\
\text { June } 7 \text { to } 8 \\
\text { June } 12 \text { to } 13 \\
\text { June } 13 \text { to } 14 \\
\text { June } 14 \text { to } 15\end{array}$ & $\begin{array}{l}49^{\circ} 55^{\prime} \mathrm{N}, 10^{\circ} 00^{\prime} \mathrm{W} \\
50^{\circ} 02^{\prime} \mathrm{N}, 10^{\circ} 22^{\prime} \mathrm{W} \\
49^{\circ} 46^{\prime} \mathrm{N}, 29^{\circ} 20^{\prime} \mathrm{W} \\
46^{\circ} 01^{\prime} \mathrm{N}, 46^{\circ} 51^{\prime} \mathrm{W} \\
41^{\circ} 51^{\prime} \mathrm{N}, 62^{\circ} 31^{\prime} \mathrm{W} \\
40^{\circ} 27^{\prime} \mathrm{N}, 73^{\circ} 50^{\prime} \mathrm{W} \\
48^{\circ} 29^{\prime} \mathrm{N}, 28^{\circ} 57^{\prime} \mathrm{W} \\
50^{\circ} 55^{\prime} \mathrm{N}, 1^{\circ} 25^{\prime} \mathrm{W} \\
49^{\circ} 40^{\prime} \mathrm{N}, 10^{\circ} 12^{\prime} \mathrm{W} \\
48^{\circ} 05^{\prime} \mathrm{N}, 28^{\circ} 30^{\prime} \mathrm{W}\end{array}$ & $\begin{array}{l}50^{\circ} 55^{\prime} \mathrm{N}, \quad 1^{\circ} 25^{\prime} \mathrm{W} \\
49^{\circ} 46^{\prime} \mathrm{N}, 29^{\circ} 20^{\prime} \mathrm{W} \\
46^{\circ} 01^{\prime} \mathrm{N}, 46^{\circ} 51^{\prime} \mathrm{W} \\
41^{\circ} 51^{\mathrm{N}}, 62^{\circ} 31^{\prime} \mathrm{W} \\
40^{\circ} 27^{\prime} \mathrm{N}, \quad 73^{\circ} 50^{\prime} \mathrm{W} \\
41^{\circ} 55^{\prime} \mathrm{N}, 62^{\circ} 15^{\prime} \mathrm{W} \\
40^{\circ} 45^{\prime} \mathrm{N}, 9^{\circ} 35^{\prime} \mathrm{W} \\
49^{\circ} 40^{\prime} \mathrm{N}, 10^{\circ} 12^{\prime} \mathrm{W} \\
48^{\circ} 05^{\prime} \mathrm{N}, 28^{\circ} 30^{\prime} \mathrm{W} \\
43^{\circ} 22^{\prime} \mathrm{N}, 48^{\circ} 38^{\prime} \mathrm{W}\end{array}$ & $\begin{aligned} & 12.6 \pm 1.3 \\
& 14.6 \pm 1.5 \\
& 9.8 \pm 1.0 \\
& 8.4 \pm 0.8 \\
& 24.0 \pm 2.4 \\
& 6.5 \pm 0.7 \\
& 7.0 \pm 0.7 \\
& 4.5 \pm 0.5 \\
& 8.1 \pm 0.8 \\
& 9.8 \pm 1.0\end{aligned}$ & $\begin{array}{r}67.4 \pm 2.0 \\
246.9 \pm 7.4 \\
149.7 \pm 4.5 \\
175.7 \pm 5.3 \\
358.5 \pm 10.8 \\
70.5 \pm 2.1 \\
105.2 \pm 3.2 \\
102.7 \pm 3.1 \\
130.1 \pm 3.9 \\
163.5 \pm 4.9\end{array}$ & $\begin{array}{r}113.5 \pm 11.4 \\
106.8 \pm 10.7 \\
63.1 \pm 6.3 \\
68.0 \pm 6.8 \\
171.3 \pm 17.1 \\
30.2 \pm 3.0 \\
36.9 \pm 3.7 \\
34.2 \pm 3.4 \\
57.0 \pm 5.7 \\
68.4 \pm 6.8\end{array}$ & $\begin{array}{r}133.2 \pm 13.3 \\
170.5 \pm 17.1 \\
110.8 \pm 11.1 \\
138.5 \pm 13.9 \\
245.0 \pm 24.5 \\
40.0 \pm 4.0 \\
82.0 \pm 8.2 \\
90.5 \pm 9.1 \\
69.8 \pm 6.9 \\
88.0 \pm 8.8\end{array}$ \\
\hline 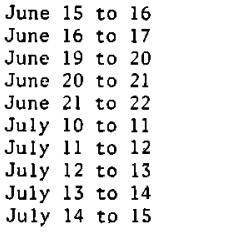 & $\begin{array}{ll}43^{\circ} 22^{\prime} \mathrm{N}, & 48^{\circ} 38^{\prime} \mathrm{W} \\
41^{\circ} 50^{\prime} \mathrm{N}, & 59^{\circ} 45^{\prime} \mathrm{W} \\
40^{\circ} 37^{\prime} \mathrm{N}, & 64^{\circ} 52^{\prime} \mathrm{W} \\
43^{\circ} 35^{\prime} \mathrm{N}, & 48^{\circ} 27^{\prime} \mathrm{W} \\
47^{\circ} 46^{\prime} \mathrm{N}, & 31^{\circ} 44^{\prime} \mathrm{W} \\
50^{\circ} 55^{\prime} \mathrm{N}, & 1^{\circ} 25^{\prime} \mathrm{W} \\
49^{\circ} 56^{\prime} \mathrm{N}, & 1^{\circ} 34^{\prime} \mathrm{W} \\
48^{\circ} 40^{\prime} \mathrm{N}, & 28^{\circ} 50^{\prime} \mathrm{W} \\
44^{\circ} 45^{\prime} \mathrm{N}, & 45^{\circ} 05^{\prime} \mathrm{W} \\
41^{\circ} 50^{\prime} \mathrm{N}, & 61^{\circ} 10^{\prime} \mathrm{W}\end{array}$ & $\begin{array}{l}41^{\circ} 50^{\prime} \mathrm{N}, 59^{\circ} 45^{\prime} \mathrm{W} \\
40^{\circ} 40^{\prime} \mathrm{N}, 73^{\circ} 50^{\prime} \mathrm{W} \\
43^{\circ} 35^{\prime} \mathrm{N}, 48^{\circ} 27^{\prime} \mathrm{W} \\
47^{\circ} 46^{\prime} \mathrm{N}, 31^{\circ} 44^{\prime} \mathrm{W} \\
49^{\circ} 38^{\prime} \mathrm{N}, 15^{\circ} 00^{\prime} \mathrm{W} \\
49^{\circ} 56^{\prime} \mathrm{N}, 10^{\circ} 34^{\prime} \mathrm{W} \\
48^{\circ} 40^{\prime} \mathrm{N}, 28^{\circ} 50^{\prime} \mathrm{W} \\
44^{\circ} 45^{\prime} \mathrm{N}, 45^{\circ} 05^{\prime} \mathrm{W} \\
41^{\circ} 50^{\prime} \mathrm{N}, 61^{\circ} 10^{\prime} \mathrm{W} \\
40^{\circ} 40^{\prime} \mathrm{N}, 73^{\circ} 50^{\prime} \mathrm{W}\end{array}$ & $\begin{aligned} & 4.8 \pm 0.5 \\
& 16.9 \pm 1.7 \\
& 4.5 \pm 0.5 \\
& 9.8 \pm 1.0 \\
& 5.3 \pm 0.5 \\
& 7.3 \pm 0.7 \\
& 11.5 \pm 1.2 \\
& 9.3 \pm 0.9 \\
& 4.8 \pm 0.5 \\
& 13.2 \pm 1.3\end{aligned}$ & $\begin{aligned} 87.3 & \pm 2.6 \\
276.9 & \pm 8.3 \\
98.6 & \pm 3.0 \\
138.1 & \pm 4.1 \\
129.9 & \pm 3.9 \\
86.6 & \pm 2.6 \\
134.5 & \pm 4.0 \\
125.4 & \pm 3.8 \\
48.8 & \pm 1.5 \\
145.7 & \pm 4.4\end{aligned}$ & $\begin{array}{r}43.1 \pm 4.3 \\
118.9 \pm 11.9 \\
47.3 \pm 4.7 \\
58.0 \pm 5.8 \\
48.3 \pm 4.8 \\
27.4 \pm 2.7 \\
56.0 \pm 5.6 \\
44.9 \pm 4.5 \\
17.3 \pm 1.7 \\
57.5 \pm 5.8\end{array}$ & $\begin{aligned} 64.7 & \pm 6.5 \\
187.6 & \pm 18.8 \\
84.6 & \pm 8.5 \\
140.4 & \pm 14.0 \\
91.9 & \pm 9.2 \\
107.2 & \pm 10.7 \\
82.1 & \pm 8.2 \\
92.1 & \pm 9.2 \\
37.3 & \pm 3.7 \\
77.0 & \pm 7.7\end{aligned}$ \\
\hline $\begin{array}{llll}\text { July } & 16 & \text { to } & 17 \\
\text { July } & 17 & \text { to } & 18 \\
\text { July } & 18 & \text { to } & 19 \\
\text { July } & 19 & \text { to } & 20 \\
\text { July } & 20 & \text { to } & 21 \\
\text { Aug. } 22 & \text { to } 23 \\
\text { Aug. } 23 & \text { to } 24 \\
\text { Aug. } 24 & \text { to } 25 \\
\text { Aug. } 25 \text { to } 26 \\
\text { Aug. } 27 \text { to } 28\end{array}$ & $\begin{array}{l}40^{\circ} 40^{\circ} \mathrm{N}, \quad 73^{\circ} 50^{\prime} \mathrm{W} \\
41^{\circ} 10^{\prime} \mathrm{N}, 64^{\circ} 50^{\prime} \mathrm{W} \\
44^{\circ} 10^{\prime} \mathrm{N}, 48^{\circ} 25^{\prime} \mathrm{W} \\
48^{\circ} 09^{\prime} \mathrm{N}, 31^{\circ} 38^{\prime} \mathrm{W} \\
49^{\circ} 47^{\prime} \mathrm{N}, 13^{\circ} 00^{\prime} \mathrm{W} \\
49^{\circ} 55^{\prime} \mathrm{N}, 9^{\circ} 05^{\prime} \mathrm{W} \\
49^{\circ} 50^{\prime} \mathrm{N}, 28^{\circ} 50^{\prime} \mathrm{W} \\
47^{\circ} 00^{\prime} \mathrm{N}, \quad 45^{\circ} 44^{\prime} \mathrm{W} \\
42^{\circ} 15^{\prime} \mathrm{N}, 61^{\circ} 25^{\prime} \mathrm{W} \\
39^{\circ} 27^{\prime} \mathrm{N}, 73^{\circ} 50^{\prime} \mathrm{W}\end{array}$ & $\begin{array}{l}41^{\circ} 10^{\prime} \mathrm{N}, 64^{\circ} 50^{\prime} \mathrm{W} \\
44^{\circ} 10^{\prime} \mathrm{N}, 48^{\circ} 25^{\prime} \mathrm{W} \\
48^{\circ} 09^{\prime} \mathrm{N}, 31^{\circ} 38^{\prime} \mathrm{W} \\
49^{\circ} 47^{\prime} \mathrm{N}, 13^{\circ} 00^{\prime} \mathrm{W} \\
50^{\circ} 55^{\prime} \mathrm{N}, 1^{\circ} 25^{\prime} \mathrm{W} \\
49^{\circ} 50^{\prime} \mathrm{N}, 28^{\circ} 50^{\prime} \mathrm{W} \\
47^{\circ} 00^{\circ} \mathrm{N}, 45^{\circ} 44^{\prime} \mathrm{W} \\
42^{\circ} 15^{\prime} \mathrm{N}, 61^{\circ} 25^{\prime} \mathrm{W} \\
39^{\circ} 27^{\prime} \mathrm{N}, 73^{\circ} 50^{\prime} \mathrm{W} \\
41^{\circ} 20^{\circ} \mathrm{N}, 64^{\circ} 30^{\prime} \mathrm{W}\end{array}$ & $\begin{array}{l}4.2 \pm 0.4 \\
8.1 \pm 0.8 \\
3.6 \pm 0.4 \\
3.1 \pm 0.3 \\
1.4 \pm 0.1 \\
2.5 \pm 0.3 \\
2.5 \pm 0.3 \\
5.1 \pm 0.5 \\
5.9 \pm 0.6\end{array}$ & $\begin{aligned} 151.0 & \pm 4.5 \\
135.9 & \pm 4.1 \\
81.3 & \pm 2.4 \\
50.8 & \pm 1.5 \\
30.4 & \pm 0.9 \\
35.4 & \pm 1.1 \\
15.1 & \pm 0.5 \\
28.3 & \pm 0.8 \\
61.2 & \pm 1.8 \\
62.6 & \pm 1.9\end{aligned}$ & $\begin{array}{r}44.0 \pm 4.4 \\
45.0 \pm 4.5 \\
22.9 \pm 2.3 \\
22.1 \pm 2.2 \\
12.2 \pm 1.2 \\
8.9 \pm 0.9 \\
3.0 \pm 0.3 \\
6.7 \pm 0.7 \\
15.4 \pm 1.5 \\
15.9 \pm 1.6\end{array}$ & $\begin{array}{l}72.7 \pm 7.3 \\
79.0 \pm 7.9 \\
45.5 \pm 4.6 \\
52.7 \pm 5.3 \\
16.7 \pm 1.7 \\
25.3 \pm 2.5 \\
27.6 \pm 2.8 \\
39.1 \pm 3.9 \\
65.9 \pm 6.6 \\
44.9 \pm 4.5\end{array}$ \\
\hline $\begin{array}{l}\text { Aug. } 28 \text { to } 29 \\
\text { Aug. } 29 \text { to } 30 \\
\text { Aug. } 30 \text { to } 31 \\
\text { Aug. } 31 \text { to } \text { Sept. } 1 \\
\text { Sept. } 7 \text { to } 8 \\
\text { Sept. } 8 \text { to } 9 \\
\text { Sept. } 10 \text { to } 11 \\
\text { Sept. } 11 \text { to } 12 \\
\text { Sept. } 12 \text { to } 13 \\
\text { Sept. } 13 \text { to } 14\end{array}$ & 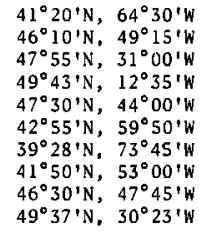 & $\begin{array}{l}46^{\circ} 10^{\prime} \mathrm{N}, 49^{\circ} 15^{\prime} \mathrm{W} \\
47^{\circ} 55^{\prime} \mathrm{N}, 31^{\circ} \mathrm{D} 0^{\prime} \mathrm{W} \\
49^{\circ} 43^{\prime} \mathrm{N}, 12^{\circ} 35^{\prime} \mathrm{W} \\
50^{\circ} 40^{\prime} \mathrm{N}, 1^{\circ} 00^{\prime} \mathrm{W} \\
42^{\circ} 55^{\prime} \mathrm{N}, 59^{\circ} 50^{\prime} \mathrm{W} \\
39^{\circ} 28^{\prime} \mathrm{N}, 73^{\circ} 45^{\prime} \mathrm{W} \\
41^{\circ} 50^{\prime} \mathrm{N}, 53^{\circ} 00^{\prime} \mathrm{W} \\
46^{\circ} 30^{\prime} \mathrm{N}, 47^{\circ} 45^{\prime} \mathrm{W} \\
49^{\circ} 37^{\prime} \mathrm{N}, 30^{\circ} 23^{\prime} \mathrm{W} \\
50^{\circ} 05^{\prime} \mathrm{N}, 11^{\circ} 10^{\prime} \mathrm{W}\end{array}$ & $\begin{array}{l}1.1 \pm 0.1 \\
3.4 \pm 0.3 \\
2.0 \pm 0.2 \\
1.4 \pm 0.1\end{array}$ & $\begin{aligned} 14.5 & \pm 0.4 \\
7.2 & \pm 0.2 \\
17.0 & \pm 0.5 \\
22.3 & \pm 0.7 \\
19.2 & \pm 0.6 \\
8.2 & \pm 0.2 \\
62.8 & \pm 1.9 \\
22.7 & \pm 0.7 \\
13.3 & \pm 0.4 \\
15.9 & \pm 0.5\end{aligned}$ & $\begin{aligned} 3.3 & \pm 0.3 \\
8.4 & \pm 0.8 \\
5.9 & \pm 0.6 \\
2.4 & \pm 0.2 \\
13.2 & \pm 1.3 \\
6.6 & \pm 0.7 \\
4.1 & \pm 0.4\end{aligned}$ & $\begin{aligned} 7.8 & \pm 0.8 \\
5.2 & \pm 0.5 \\
29.6 & \pm 3.0 \\
48.4 & \pm 4.8 \\
33.2 & \pm 3.3 \\
5.2 & \pm 0.5 \\
89.1 & \pm 8.9 \\
57.6 & \pm 5.8 \\
26.0 & \pm 2.6 \\
23.4 & \pm 2.8\end{aligned}$ \\
\hline $\begin{array}{l}\text { Sept. } 14 \text { to } 15 \\
\text { Sept. } 21 \text { to } 22 \\
\text { Sept. } 24 \text { to } 25 \\
\text { Sept. } 25 \text { to } 26 \\
\text { Oct. } 17 \text { to } 18 \\
\text { oct. } 18 \text { to } 19 \\
\text { Oct. } 19 \text { to } 20 \\
\text { Oct. } 20 \text { to } 21 \\
\text { Oct. } 22 \text { to } 23 \\
\text { Oct. } 23 \text { to } 24 \\
\text { Oct. } 24 \text { to } 25 \\
\text { Oct. } 25 \text { to } 26\end{array}$ & 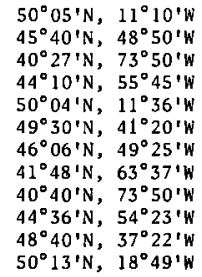 & $\begin{array}{l}50^{\circ} 40^{\prime} \mathrm{N}, 0^{\circ} 55^{\prime} \mathrm{W} \\
42^{\circ} 20^{\prime} \mathrm{N}, 61^{\circ} 05^{\prime} \mathrm{W} \\
44^{\circ} 10^{\prime} \mathrm{N}, 55^{\circ} 45^{\prime} \mathrm{W} \\
48^{\circ} 30^{\prime} \mathrm{N}, 39^{\circ} 00^{\prime} \mathrm{W} \\
49^{\circ} 30^{\prime} \mathrm{N}, 41^{\circ} 20^{\prime} \mathrm{W} \\
46^{\circ} 06^{\prime} \mathrm{N}, 49^{\circ} 25^{\prime} \mathrm{W} \\
41^{\circ} 48^{\prime} \mathrm{N}, 63^{\circ} 37^{\prime} \mathrm{W} \\
40^{\circ} 40^{\prime} \mathrm{N}, 73^{\circ} 50^{\prime} \mathrm{W} \\
44^{\circ} 36^{\prime} \mathrm{N}, 54^{\circ} 23^{\prime} \mathrm{W} \\
48^{\circ} 40^{\prime} \mathrm{N}, 37^{\circ} 22^{\prime} \mathrm{W} \\
50^{\circ} 13^{\prime} \mathrm{N}, 18^{\circ} 49^{\prime} \mathrm{W} \\
50^{\circ} 55^{\prime} \mathrm{N}, 1^{\circ} 25^{\prime} \mathrm{W}\end{array}$ & $\begin{array}{l}4.5 \pm 0.5 \\
1.1 \pm 0.1 \\
1.7 \pm 0.2 \\
1.4 \pm 0.1 \\
3.4 \pm 0.3 \\
3.9 \pm 0.4 \\
3.6 \pm 0.4\end{array}$ & $\begin{array}{r}9.7 \pm 0.3 \\
50.4 \pm 1.5 \\
17.3 \pm 0.5 \\
25.4 \pm 0.8 \\
22.5 \pm 0.7 \\
27.2 \pm 0.8 \\
41.1 \pm 1.2 \\
44.1 \pm 1.3 \\
53.9 \pm 1.6 \\
35.8 \pm 1.1 \\
27.4 \pm 0.8 \\
10.7 \pm 0.3\end{array}$ & $\begin{aligned} 15.1 & \pm 1.5 \\
4.0 & \pm 0.4 \\
7.2 & \pm 0.7 \\
4.6 & \pm 0.5 \\
4.7 & \pm 0.5 \\
5.4 & \pm 0.5 \\
7.5 & \pm 0.8 \\
6.2 & \pm 0.6\end{aligned}$ & $\begin{aligned} 15.9 & \pm 1.6 \\
100.8 & \pm 10.1 \\
33.2 & \pm 3.3 \\
25.2 & \pm 2.5 \\
53.9 & \pm 5.4 \\
43.0 & \pm 4.3 \\
75.8 & \pm 7.6 \\
64.4 & \pm 6.4 \\
100.1 & \pm 10.0 \\
83.1 & \pm 8.3 \\
83.1 & \pm 8.3 \\
36.2 & \pm 3.6\end{aligned}$ \\
\hline
\end{tabular}

*All data corrected to date of collection. 


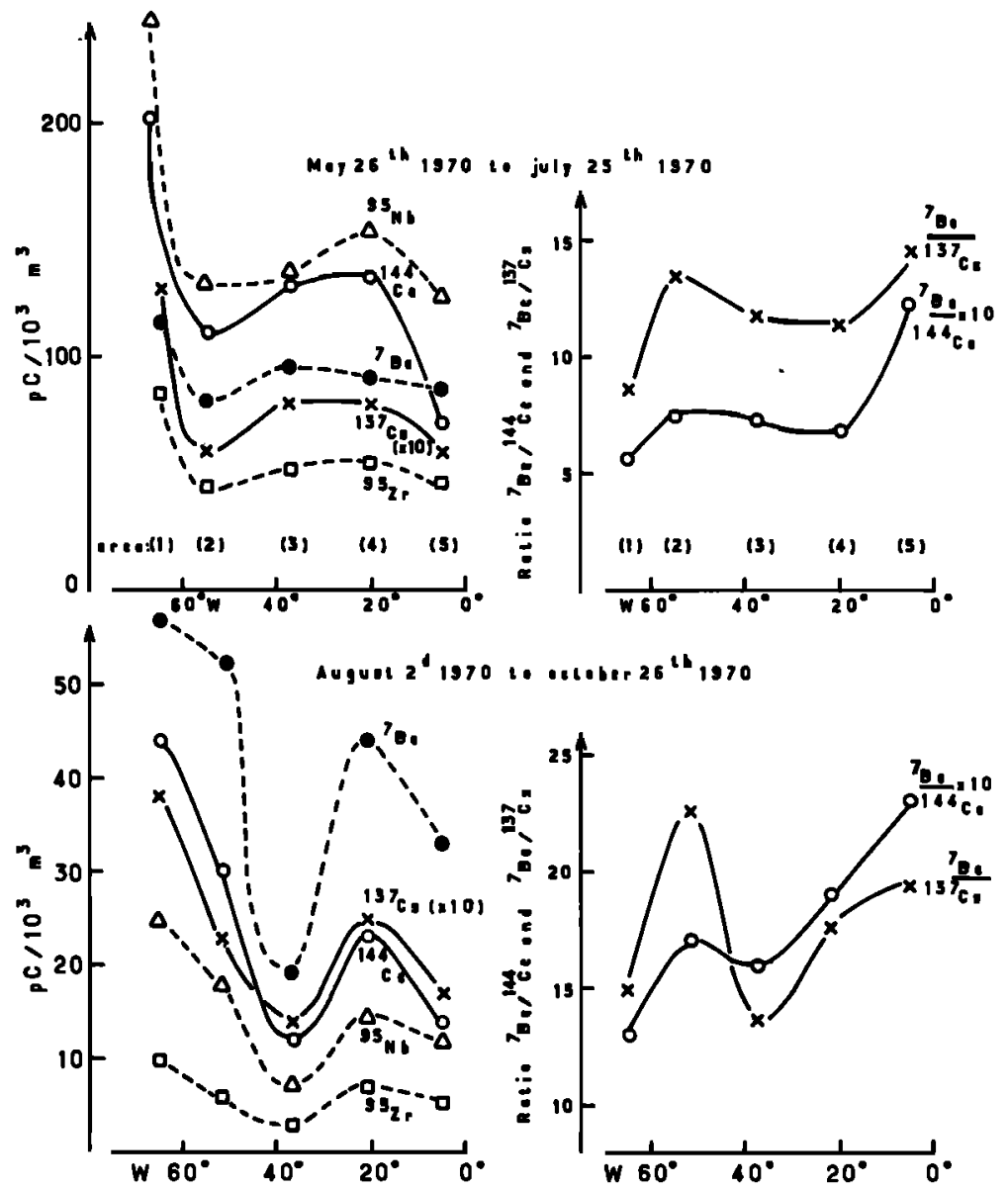

Fig. 3. Variation with longitude of aerosol radioactivity (oceanic samples collected between Le Havre and New York aboard the ship France).

However, this phenomenon is not observable in autumn, when stratospheric injections are very poor. Therefore, it now seems possible that the origin of the longitudinal inhomogeneities is not only due to the jet stream standing waves. Anyway, such inhomogeneities have to be taken into account for all global estimations of radioactive debris deposition.

Acknowledgment. We are very grateful to Dr. Hoang Chi Trach for the sampling aboard Charcot the Cie Gle Transatlantique, the crew of France for the sampling, and Dr. J. C. Philippot for his cooperation in $\gamma$ spectrometry analyses.

\section{REFERENCES}

Lambert, G., B. Ardouin, G. Polian, and J. Sanak, Natural radioactivity balance in the atmosphere of the southern hemisphere, paper presented at the second International Symposium on Natural Radiation in the Environment, Houston, Texas, August 7-11, 1972.

Nguyen Ba Cuong, and G. Lambert, Geographic variations of the large-scale vertical exchange speed, J. Geophys. Res., 75, 2877-2884, 1970.

Rossby, C. G., On the general circulation in middle latitudes, Bull. Amer. Meteorol. Soc., 28, 255-280, 1947.

Volchok, H. L., and B. Krajewski, Radionuclides and lead in surface air, Fallout program quarterly report, Rep. 249, C1-C102, HASL, Washington, D. C., 1972.

(Received February 21, 1973; revised March 28, 1973.) 